\title{
Catalysis and Atom Probe Tomography: Recent Progresses and Future Developments towards the Analysis of Nanoporous Samples
}

\author{
Cédric Barroo ${ }^{1}$, Andrew P. Magyar ${ }^{2}$ and David C. Bell ${ }^{1,2}$ \\ 1. School of Engineering and Applied Sciences, Harvard University, Cambridge MA USA \\ 2. Center for Nanoscale Systems, Harvard University, Cambridge MA USA
}

Since the infancy of microscopy and surface science, many advanced techniques have been utilized to better understand catalytic systems. Previous studies focus either on the catalytic reaction (reactivity and selectivity) or on the catalyst itself, focusing on structural/morphological reconstructions as well as changes in the chemical composition of the catalyst. Atom probe tomography (APT) is a powerful tool for the characterization of both the three-dimensional structure and composition of catalysts at the atomic-scale. Alloys catalysts are of particular interest in applied catalysis because the synergistic effects between the different metals can drastically influence their activity as compared to their constituting pure metals [1]. At the microscopic scale, the surface composition of the very first atomic layers can differ from that of the bulk. The surface composition can be modified via physicochemical treatments, yielding different catalytic behaviours, which are highly dependent on the composition of the alloy, the temperature and duration of treatments, the crystallographic orientation, and the presence of surface or bulk defects. Among others, surface segregation/depletion, core-shell structure and formation of nano-islands can be studied by APT and can inform the nanoscale engineering of catalyst surface compositions (see [2] and references therein). For such studies, a needled shape catalyst is used, which can then be directly measured via APT. More recently, developments have been made regarding the sample preparation allowing the analysis of more realistic catalysts by atom probe tomography. Conventional tip samples used in APT can be used as a support for nanoparticles of catalysts using an electrophoresis method [3] and this method has been successfully used for the analysis of Pt, Pt-Co, Ir@Pt and Ag@Pd nanoparticles [1,3]. Eventually, embedding methods and FIB tip preparation can be used to study well-dispersed nanoparticles [4].

Current research focuses on the use of nanostructured materials, such as powders and nanoporous catalysts, for energy applications. As an example, nanoporous gold catalysts (npAu) exhibit a high activity and selectivity for alcohol oxidation reactions, which has been attributed to the presence of traces of silver, originating from the sample preparation. The systematic study of such a catalyst requires a control of its microstructure. However, the very morphology of this class of catalysts and the presence of pores make the APT analysis and the reconstruction impossible via traditional techniques. The sample preparation thus requires new developments. A glass micro-encapsulation technique has also been developed to study nanoparticles and powders by APT [5] and provides a rapid, robust, and inexpensive way to analyze nano- or micro-particles using LEAP, however the amorphous nature of the glass can also present challenges for this approach.

The present study reports the analysis of nanoporous gold with traces of silver. This has been made possible by the combination of SEM, TEM and APT analysis. To study those catalysts, different methodologies are used to fill the pores and then allow the analysis by atom probe: atomic layer deposition (ALD) (an example of APT analysis of Al-doped ZnO (AZO) film deposited by ALD is presented Fig1a), e-beam evaporation, and in situ deposition in the FIB, prior to the regular lift-out method. The npAu samples are studied at different stages of the catalytic reaction to trace the evolution 
of the catalyst's structure from its initial state through its final form. The stages corresponds to 1) the initial catalyst prior to any treatment, 2) the catalyst after ozone pre-treatment, 3) the sample right after the first moment of the reaction, and 4) the active catalyst, namely the catalyst after establishment of a steady state regime of conversion.

The combined approach can allow correlation of the nano-structure of a nanoporous gold matrix, derived from TEM with the 3D nano-structure of the same system obtained from APT both before and after exposure to reaction conditions. These results directly indicate a coarsening of the nanogold matrix due to the reaction event (Fig1b). The behavior of the silver atoms within the gold backbone is also studied in details, and the formation of $\mathrm{Au}-\mathrm{Ag}$ alloy is supposed to be responsible of the activity of the catalyst. [6]

\section{References:}

[1] K. Tedsree, et al., Nature Nanotech. 6 (2011) 302

[2] C. Barroo, P. A. J. Bagot, G. D. W. Smith, T. Visart de Bocarmé, "Investigating Nano-structured Catalysts at the Atomic scale by Field Ion Microscopy and Atom Probe Tomography", in Atomically-

Precise Methods for Synthesis of Solid Catalysts; RSC Catalysis Series, 2015, pp. 248-295

[3] T. Li, et al., ACS Catal. 4 (2014) 695

[4] P. Felfer, et al., Angew. Chem. 126 (2014) 11372

[5] D. C. Bell, et al., Microscopy and Microanalysis, 19 [S2] (2013) 954

[6] This work was supported as part of the Integrated Mesoscale Architectures for Sustainable Catalysis - IMASC, an Energy Frontier Research Center funded by the U.S. Department of Energy, Office of Science, Basic Energy Sciences under Award \# DE-SC0012573. C.B. acknowledges postdoctoral fellowships through the Belgian American Educational Foundation (BAEF) as well as WallonieBruxelles International (Excellence grant WBI.WORLD) foundations.
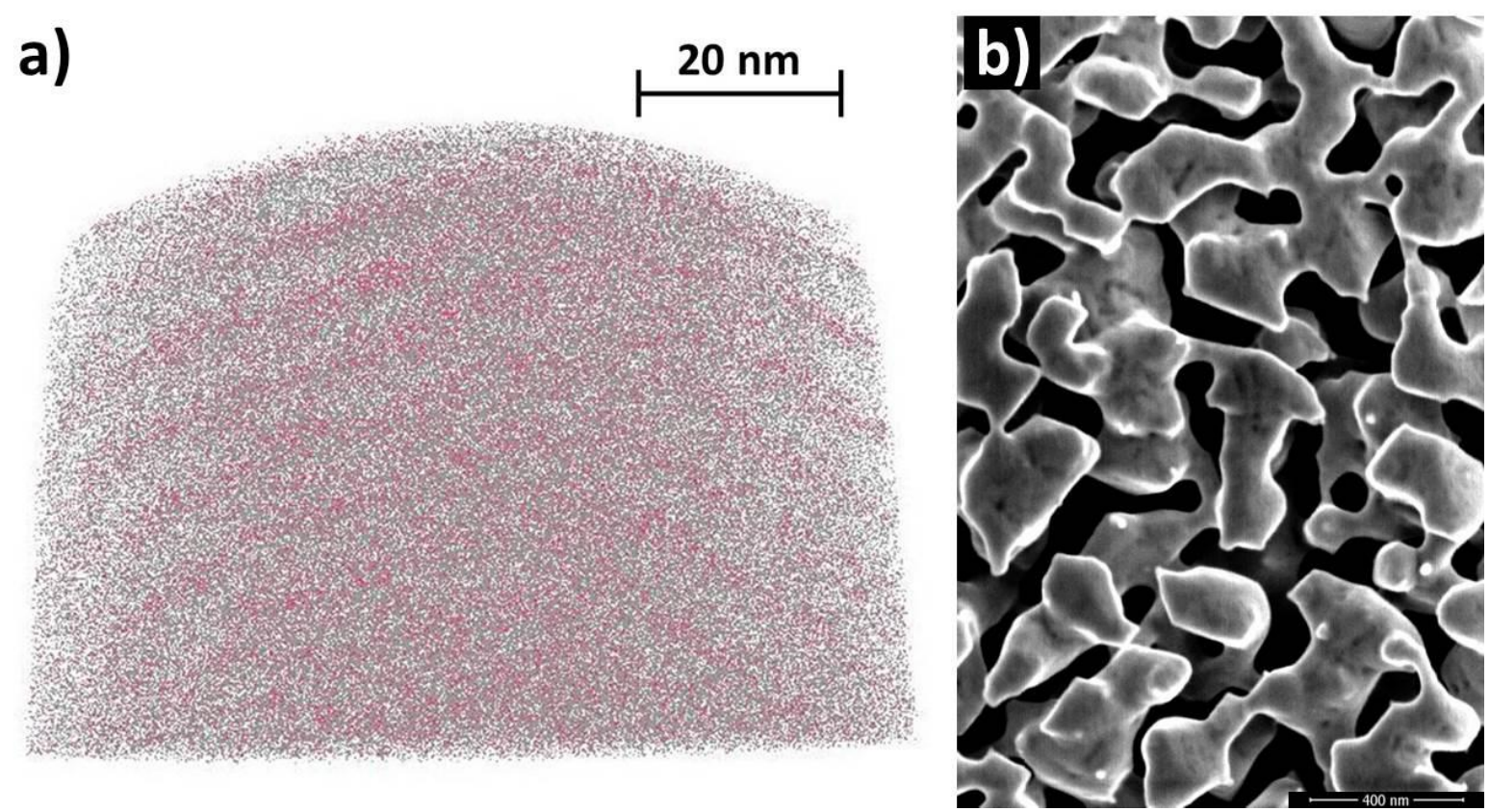

Figure 1. a) 3D reconstruction of Al-doped $\mathrm{ZnO}(\mathrm{AZO})$ deposited by atomic layer deposition on a presharped APT (red balls corresponds to Al atoms, and grey to $\mathrm{Zn}$ atoms) - b) SEM evidence of coarsening of npAu after reaction (scale bar: $400 \mathrm{~nm}$ ). 